Fortschr. Neurol. Psychiat. 50 (1982) 133-138

(C) Georg Thieme Verlag Stuttgart - New York

\title{
Liquorproteine bei der Neuromyelitis optica
}

\author{
K.-H. Krause, P. Berlit, H.P. Schmitt, R. v. Kummer \\ Neurologische Klinik (Direktor: Prof. Dr. H. Gänshirt) und Neuropathologisches Institut \\ (Direktor: Prof. Dr. G. Ule) der Universität Heidelberg
}

\begin{abstract}
CSF-proteins in nouromyelitis optice
Two cases of neuromyelitis optica are reported. Both patients had a very high CSF-protein-level. One patient died after a period of six months and his brain was studied neuropathologicelly. The other patient survived with heavy residual symptoms; in his CSF as well as in his serum clearly increased IgA - and IgM - levels were found. The CSF.findings seem to be helpful in the differential diagnosis of multiple sclerosis and neuromyelitis optica.
\end{abstract}

\section{Zusammenfassung}

Es werden zwei Fälle von Neuromyelitis optica beschrieben, die jeweils eine hochgradige Erhöhung der Proteine im Liquor aufwiesen. Während ein Patient nach 6monatigem Krankheitsverlauf starb und pathologisch-anatomisch untersucht werden konnte, überlebte der andere Patient mit erheblichen Residuen. Dieser Fall zeichnete sich dadurch aus, daß deutlich erhöhte IgA- und IgM-Werte sowohl im Liquor als auch im Serum vorlagen. Es wird diskutiert, ob die gefundenen Liquorveränderungen möglicherweise eine Abgrenzung entsprechender Fälle gegenüber der multiplen Sklerose zulassen.

Die nosologische Einordnung des von Devic (5) und Gault (7) beschriebenen Syndroms der Neuromyelitis optica (N.o.) war stets kontrovers. Neben der Annahme einer eigenständigen nosologischen Entität $(1,2,8,10,13,15,17)$ wurde ausgehend von der klinischen Symptomatologie - immer wieder das Vorliegen einer akuten Verlaufsform der Enze phalomyelitis disseminata diskutiert $(6,9,11,14,18,23$, 27, 28). Cloys und Netsky (3) fassen au fgrund dieser Schwierigkeiten die Neuromyelitis optica lediglich als Symptomkombination auf, die im Rahmen verschiedener demyelinisierender Erkrankungen wie multipler Sklerose, diffuser Sklerose und postinfektiöser Enzephalomyelitis auftreten kann. Oppenheimer (21) unterscheidet aus neuropathologischer Sicht vier Formen der Neuromyelitis optica: 1. schwere Demyelinisierung mit Nekrose eines Rückenmarksabschnittes, 2. zusätzlich hierzu Plaques wie bei der akuten multiplen Sklerose in anderen Arealen des Zentralnervensystems oder Läsionen vom Schilderschen Typ in den Hemisphären, 3. Läsionen wie bei akuter oder chronischer MS ohne Nekrose und 4. Läsionen wie bei der perivenösen Enzephalomyelitis. Die Typen 1 und 2 unterscheiden sich durch das Auftreten von Gewebsnekrosen grundsätzlich von den histopathologischen Befunden bei der klassischen chronischen MS. Dies ist der wesentliche Grund, weshalb von vielen maßgeblichen Autoren die Zugehörigkeit dieser Krankheitsbilder zum Spektrum der MS bestritten wurde.

Ähnlich kontrovers wie die Ansichten zur Nosologie sind die Angaben hinsichtlich der Liquorbefunde. Teilweise wird ein Unterschied zwischen Neuromyelitis optica und Encephalomyelitis disseminata überhaupt negiert $(2,30)$, teils werden diskrete Eiweißerhöhungen vermerkt (3); andererseits finden sich aber auch Mitteilungen über massive Eiweißerhöhungen $(12,13)$.

Im folgenden sollen zwei Fälle mit N.o. beschrieben werden, die pathologische Liquorbefunde aufwiesen, wie sie bei multipler Sklerose nicht zu beobachten sind.

\section{Kasuistik}

\section{Fall 1:}

Frau WP., 34 Jahre, stationäre Aufnahme am 18.4.79. Keine ernsthaften Vorerkrankungen bekannt. Im Alter von $15 \mathrm{Jahren}$ litt Frau W.P. unter einer Retrobulbärneuritis rechts mit nachfolgender Optikusatrophie und einem Restvisus von 1/25 auf diesem Auge. 6 Jahre später traten Sehstörungen auch auf dem linken Auge auf; der Nervus opticus links atrophierte ebenfalls. Seit dieser Zeit kann die Patientin links nur noch hell-dunkel unterscheiden. Mit 24 Jahren fiel eine akute Hörminderung auf, die bei einer HNO-ärztlichen Untersuchung als zentral bedingt gedeutet wurde. Seit 1976 litt Frau WP. wiederholt unter Schwächegefühlen in den Armen, 2 Jahre später bemerkte sie eine Ungeschicklichkeit und Schwäche im rechten Bein sowie eine Zunahme der Kraftlosigkeit in den Armen, außerdem traten kribbelnde Mißempfindungen im Bereich beider Unterarme sowie des rechten Unterschenkels auf. Seit Anfang 1979 hatte sich der Zustand der Patientin rapide verschlechtert, seit Februar des Jahres war selbständiges Gehen nicht mehr möglich gewesen.

Bei der neurologischen Untersuchung fand sich eine hochgradige Sehstörung mit Reduktion des Visus auf Hell-dunkelUnterscheidung links und schwaches Erkennen von bewegten Gegenständen rechts bei atrophischen Sehnervenpapillen; weiterhin bestand eine links etwas stärker als rechts ausgeprägte mäßige Innenohrschwerhörigkeit. Im Myotom C 5 und C 6 fanden sich beidseits deutliche schlaffe Paresen mit Atrophie, Minderung des Muskeltonus bei ausgefallenem Brachioradialis- und nur sehr schwach auslösbarem Bizeps- und Trizepsreflex beidseits. Unterhalb waren Zeichen einer deutlichen rechtsbeton ten spastischen Tetraparese mit rechtsbe- 
tontem, sehr lebhaften Knipsreflex und Trömnerschen Zeichen, nur sehr schwach, im rechten mittleren und unteren Quadranten nicht auslösbaren Bauchhautreflexen und gesteigerten Beineigenreflexen mit unerschöpflichem Fußklonus rechts nachzuweisen. Das Babinskische Zeichen war rechts positiv, der Rossolimosche Reflex rechts lebhafter als links. Während der linke Arm aktiv noch kurze Zeit hochgehalten werden konnte, war die proximal betonte Lähmung am rechten Arm so ausgeprägt, daß eine Elevation nicht möglich war. Die Rumpfmuskulatur war erheblich paretisch - die Patientin konnte sich mit eigener Kraft aus liegender Position nicht aufrichten. An den Beinen bestand rechtsbetont eine mittelgradige Parese sämtlicher Muskeln mit deutlicher Bevorzugung der Hüftbeuger sowie der Fuß- und Zehenstrecker. Durch immer wieder beim Versuch der Willkürinnervation v.a. in die proximale Beinmuskulatur einschießende Spasmen war die Patientin so behindert, daß Gehversuche scheiterten. Sensibel bestand ab $\mathrm{C} 4$ beidseits eine Störung aller Empfindungsqualitäten, wobei die epikritische Funktion an den oberen Extremitäten stärker betroffen war als an den unteren. An Armen und Rumpf fand sich eine Analgesie, an den Beinen dagegen eher eine Hyperalgesie. Die Temperatursinnstörung

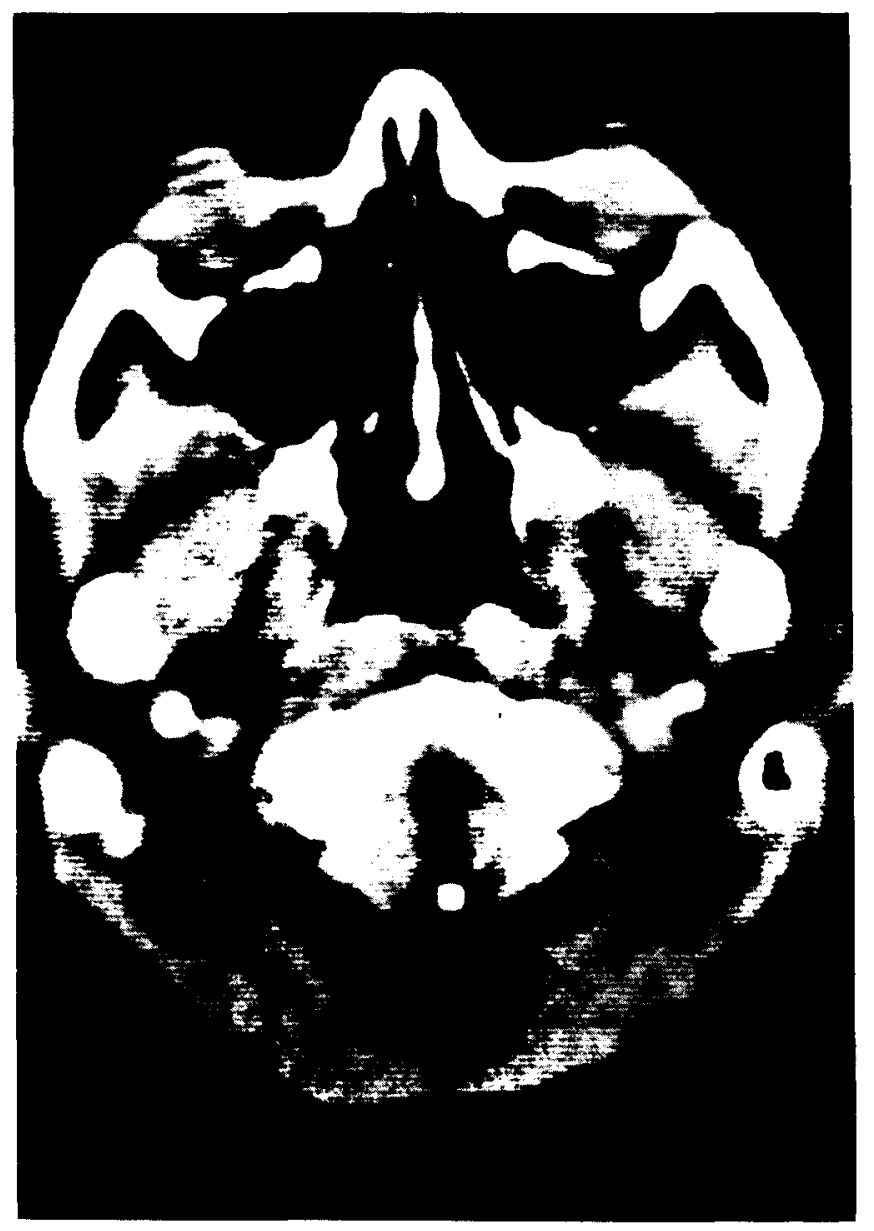

Abb. 1 Computertomogramm des oberen Halsmarkes ohne Kontrastmittel (Fall 1). Die Transversalschicht in Höhe C 1 zeigt eine zentral im Mark gelegene hantelförmige Aufhellung (mit Meßpunkt). Für die Durchführung der Untersuchung und Überlassung des Bildes danken wir Herrn Prof. Dr. G. van Kaick, Deutsches Krebsforschungszentrum, Heidelberg war am Rumpf, wo eine Thermanästhesie bestand, deutlich stärker ausgeprägt als an den Extremitäten. Es fand sich eine komplette Blasenlähmung bei weitgehend intakter Mastdarmfunktion.

Röntgenaufnahmen des Schädels, des kranio-zervikalen Úbergangs und der HWS waren unauffällig. Während ein Computertomogramm des Schädels ebenfalls unauffällige Verhältnisse zeigte, kam im CT des Halsmarks eine hantelförmige ausgedehnte Zone mit verminderter Dichte im Sinne einer Nekrose bzw. einer Zyste zur Darstellung, die sich bei Kontrastmittelgabe nicht anreicherte (Abb. 1).

Das Luftmyelogramm zeigte im oberen Abschnitt des Zervikalmarks eine zunehmende Verschmälerung in der Luftsäule. Bei der Patientin wurde viermal lumbal Liquor entnommen (Punktion [P] 1 am 20.4., P 2 am 27.4., P 3 am 9.5. und $P 4$ am 6.6.). Die Gesamteiweißmenge war bei allen Punktionen deutlich erhöht (P 1:600, P 2: 960, P 3: 648 und P 4: $432 \mathrm{mg} \%$ ) mit jeweils deutlicher Erhöhung des GlobulinAlbumin-Quotienten $>1$. Bei drei Liquorentnahmen wurden mit dem Verfahren der radialen Immunodiffusion die Immunglobuline bestimmt: P 1: IgG 354, IgA 79, IgM 34,9 mg\%, P 3: IgG 219, IgA 77,5, IgM 23,3 mg\%, P 4: IgG 179, IgA 50, IgM 6,7 mg\%. Die Zellzahl im Liquor zeigte Werte von $26 / 3$ (P 3) über 69/3 (P 4) und 264/3 (P 1) bis zu $2538 / 3$ (P 2) mit jeweils überwiegend Lymphozy ten. Sämtliche Untersuchungen auf Keime oder Antikörpertiter im Liquor waren negativ.

Bei mehrfachen Untersuchungen der Proteine im Blut fanden sich konstant normale Werte hinsichtlich des Gesamtproteins und de: Eiweißzusammensetzung. Die mit Hilfe der Radioimmundiffusion bestimmten Immunglobuline zeigten für IgG eher niedrige (785-820 mg\%), für IgA (368-421) und IgM (277-286) dagegen leicht erhöhte Werte.

\section{Klinischer Verlauf}

Unter Cortisontherapie (bis zu $80 \mathrm{mg}$ Solu-Decortilen über mehrere Wochen), antispastischer Medikation (Lioresal) und intensiver krankengymnastischer Therapie kam es während des 9wöchigen Klinikaufenthaltes nur zu einer diskreten Besserung. Durch das Ausbleiben der Spasmen in den unteren Extremitäten war die Patientin bei der Entlassung immerhin in der Lage, sich mit Hilfe von Stöcken fortzubewegen. Die Kraft- und Sensibilitätsstörung an den Armen und Beinen bestand unverändert, am Rumpf waren eindeutige Sensibilitätsstörungen nur noch ab D 11 nachzuweisen. Die Miktion war nach 3 Wochen des stationären Aufenthaltes wieder spontan möglich, der Restharn betrug bei Entlassung aber immer noch $870 \mathrm{ml}$.

\section{Fall 2:}

Herr H.H., 37 Jahre; stationäre Aufnahme am 2.11.80. Frühere Anamnese und Familienanamnese unauffallig. Wegen einer am 20.6.80 aufgetretenen Retrobulbärneuritis links mehrwöchige Behandlung in einem auswärtigen Krankenhaus ohne wesentliche Besserung. Am 1.11.80 plötzliche Schwäche der rechten Körperhälfte.

Bei der neurologischen Untersuchung fand sich eine Abblassung der Sehnervenpapille links mit Visusminderung des lin. 
ken Auges und verzögerter Pupillenreaktion auf Licht. Es bestand eine leichte sensomotorische Hemiparese rechts mit gesteigerten Muskeleigenreflexen; die Bauchhautreflexe waren beidseits rasch erschöpflich, es lag eine stumme Sohle beidseits vor. Der Restharn betrug $60 \mathrm{ml}$.

Der am Aufnahmetag lumbal entnommene Liquor enthielt $1 / 3$ Zellen bei einem Gesamteiweiß von $67,2 \mathrm{mg} \%$ (Globuline 21,6, Albumine 45,6 mg\%); Erhöhung des IgA auf 0,65 $\mathrm{mg} \%$ bei unauffälligem IgG und IgM. Sämtliche bakteriologischen und virologischen Untersuchungen im Liquor waren negativ. Im Serum waren die Eiweiß- und Immunelektrophorese regelrecht.

Unter der Verdach tsdiagnose einer Encephalomyelitis disseminata erfolgte eine Infusionsbehandlung mit ACTH $(1 \mathrm{mg} / \mathrm{d})$. Am 6.11.80 kam es zu einer Harnverhal tung, die das Einlegen eines Dauerkatheters notwendig machte. Am 8.11.80 trat zweimalig ein plötzlicher Atemstillstand auf, es erfolgten Intubation und die Verlegung auf die Beatmungsstation der Medizinischen Universitätsklinik. Dort wurde eine hochdosierte Kortikoidbehandlung eingeleitet. Nachdem wiederholte Versuche, den Patienten spontan atmen zu lassen, scheiterten, wurde ein Tracheostoma zur Dauerbeatmung angelegt. Innerhalb weniger Tage entwickelte sich ein hohes Querschnittssyndrom. Bei der neurologischen Nachuntersuchung am 20.11.80 fand sich eine Optikusatrophie links mit aufgehobener direkter Reaktion der Pupille bei ausreichender konsensueller Lichtreaktion. Es bestand ein Querschnittssyndrom bei C 2 mit kompletter Atemlähmung. Der Muskeltonus an allen Extremitäten war schlaff, es waren lediglich der Trizepsreflex und der Quadrizepsreflex - jeweils rechts lebhafter als links - auslösbar. Der lumbal entnommene Liquor war bei der Entnahme klar, gerann jedoch sofort im Röhrchen, so daß eine Zellzahlbestimmung nicht möglich war. Im Überstand betrug der Gesamteiweißwert $240 \mathrm{mg} \%$ (Globuline 64,2 $\mathrm{mg} \%$, Albumine 172,8 mg\%). Aufgrund des klinischen Bildes wurde die Diagnose einer Neuromyelitis optica gestellt. Wiederholt auftretende Pneumonien machten eine antibiotische Kombinationsbehandlung notwendig. Anfang Dezember 1980 bestand eine schlaffe Tetraplegie ohne auslösbare Muskeleigenreflexe bei stummer Sohle beidseits. Die linke Pupille war bei deutlicher Optikusatrophie weit und lichtstarr. Der am 11.12.80 entnommene Liquor war bei Entnahme $x$ an thochrom und flockte sofort aus, so daß auf eine genaue Eiweißbestimmung verzichtet wurde. Die Serumelektrophorese zeigte unauffällige Werte.

Am 3.1.81 kam es nach einem Blutdruckabfall infolge eines Herzstillstandes zum Exitus.

Pathologisch-anatomisch (S. Nr. 14/81, Institut für Allgemeine Pathologie und Pathologische Anatomie und Institut für Neuropathologie der Universität Heidelberg) fanden sich bei der allgemeinen Sektion der Brust- und Bauchhöhle als wesentliche Befunde eine pseudomembranöse Tracheitis mit schleimiger Bronchitis bei Zustand nach Tracheotomie und reizlosem Tracheostoma. Die Extremitäten zeigten eine deutliche Umfangsreduktion durch eine Verschmächtigung der Muskulatur.

Die Schädelhöhle bot keine auffallenden pathologisch-anatomischen Veränderungen; das Hirngewicht lag mit $1650 \mathrm{~g}$ deutlich über dem Durchschnitt für männliche Individuen im mittleren Lebensalter (Norm um $1400 \mathrm{~g}$ ) und ließ die
Zeichen eines Hirnödems mit intrakranieller Raumforderung erkennen.

Auf Schnitten durch Groß- und Kleinhirn sah man bereits makroskopisch multiple fleckförmige, leicht graustichige Herde von variabler Größe in der Marksubstanz, wobei teilweise die Gewebskontinuität gewahrt war, teilweise aber auch mikrozystische Kontinuitätsunterbrechungen darauf hindeuteten, daß hier ein stellenweise bis zur kompletten Nekrose führender krankhafter Prozeß vorlag. Bei den anderen Herden ließ schon der makroskopische Aspekt eine Demyelinisierung vermuten (Abb. 2). Auf parieto-okzipitalen Frontalschnitten zeigte ein links periventrikulär um die Spitze des Hinterhomes der Seitenventrikel ausgedehnter Markherd auf Ubersichtsschnitten im Markscheidenpräparat bereits mit bloßem Auge eine deutliche konzentrische Schichtung (Abb. 2a), die an das typische Bild bei Balóscher Krankheit erinnerte. Der Herd durchsetzte ausgedehnte Anteile der Gratioletschen Sehstrahlung. Das Halsmark erschien auf den Querschnitten makroskopisch aufgetrieben bei undeutlicher Zeichnung der Schnittfläche, auf welcher weiße und graue Substanz kaum voneinander abgegrenzt erschienen. Histologische Markscheidenpräparate ließen bereits mit bloßem Auge eine massive Schädigung des Halsmarkes erkennen, in dem sich praktisch keine Marksubstanz mehr färberisch dargestellt hatte (Abb. 2c, g). Dies stand in deutlichem Kontrast zu den gehörig erscheinenden Schnitten vom mittleren Brustund Lendenmark (Abb. 2c).

Mikroskopisch zeigte sich, daß neben dem subtotalen Markscheidenausfall mit erheblicher Gliose und einzelnen Makrophagen im Halsmark auch die Axone, wenngleich bei weitem nicht in demselben Umfange, Schädigungszeichen, vor allem in Form von floriden Axondegenerationen, boten. Aus der Diskrepanz zwischen dem Ausmaß der Entmarkung und dem der Axonschädigung ließ sich eindeutig die Demyelinisierung als dominierender Prozeß identifizieren. Gleiches galt im Prinzip für die verschiedenen Herde im Marklager von Groß- und Kleinhirn, die teils reine Entmarkungsphänomene, teils aber auch komplette Nekrosen mit kleinzystischer Gewebseinschmelzung zeigten. In den Nervi und Tractus optici waren diskontinuierliche Markschattenherde mit noch diskreten Markabbauprodukten nachweisbar (Abb. 2d, e).

Feingeweblich war in den meisten zerebralen Herden eine ausgeprägte Gliose aus mikroglialen und zytoplasmareichen astrozytären Elementen zu sehen. Eine fasergliotische Vernarbung sah man nur stellenweise in spärlichen Ansätzen; sie erreichte niemals ein Ausmaß, wie es bei klassischen älteren Entmarkungsherden, z.B. bei multipler Sklerose, üblich ist.

Entzündliche Erscheinungen waren nur in sehr geringem Umfange, in Form gelegentlicher schütterer perivaskulärer Rundzelleninfiltrate, nachzuweisen, wobei allerdings die intensive Steroidbehandlung des Patienten in Rechnung gestellt werden muß.

Im Nervus peronaeus fanden sich z.T. ausgeprägte floride Demyelinisierungserscheinungen mit Markballenbildung, ohne adäquate Ausfälle im Axonbild. Dementsprechend boten die Beinmuskeln das Bild einer diffusen (,Inaktivitäts"-) A trophie ohne eindeutige neurogen-atrophische Komponente. 

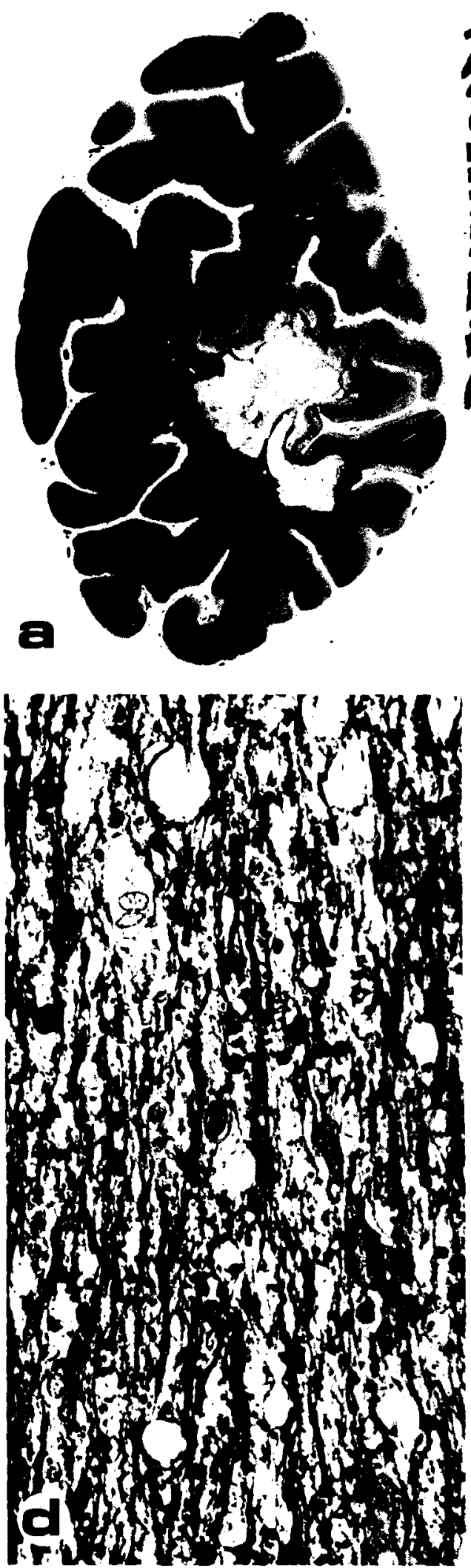
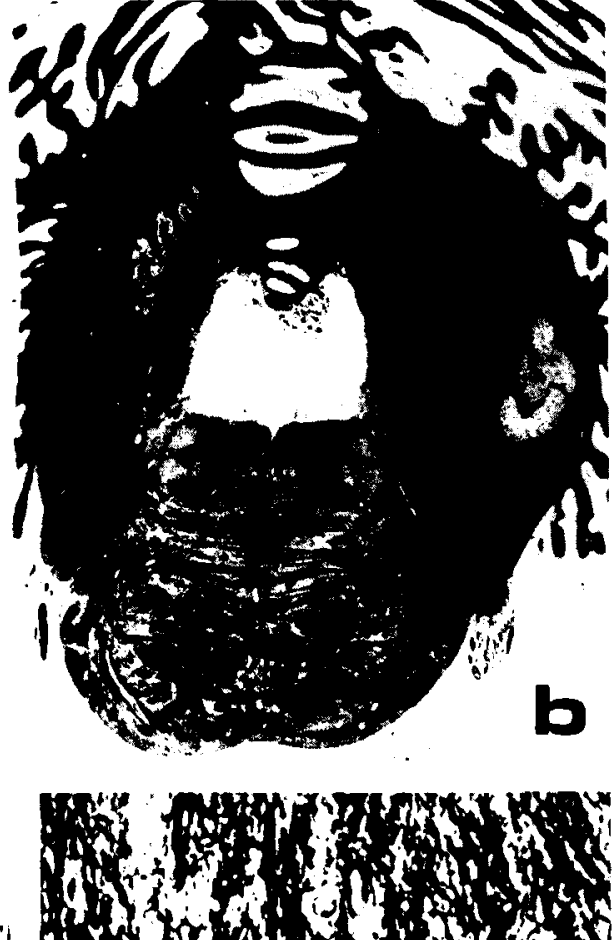

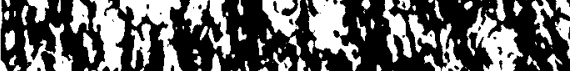

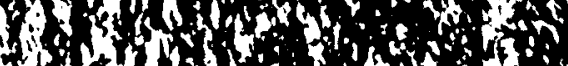

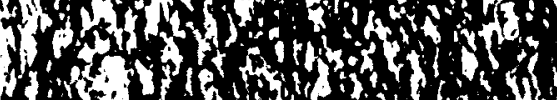

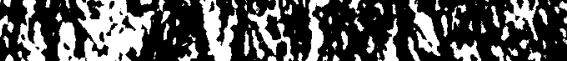

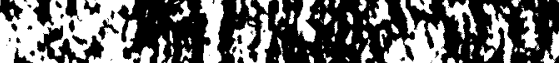
ivis

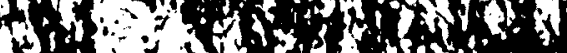

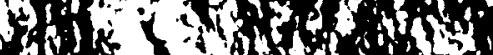
4 fo

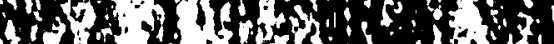
of

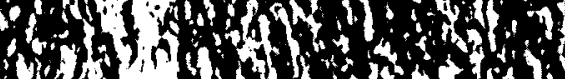

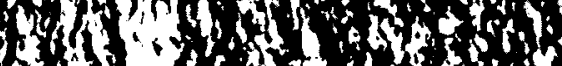

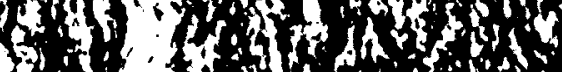

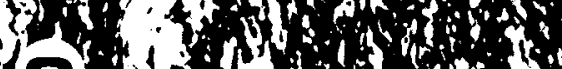

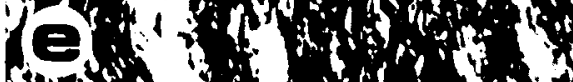

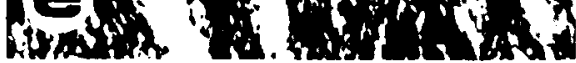
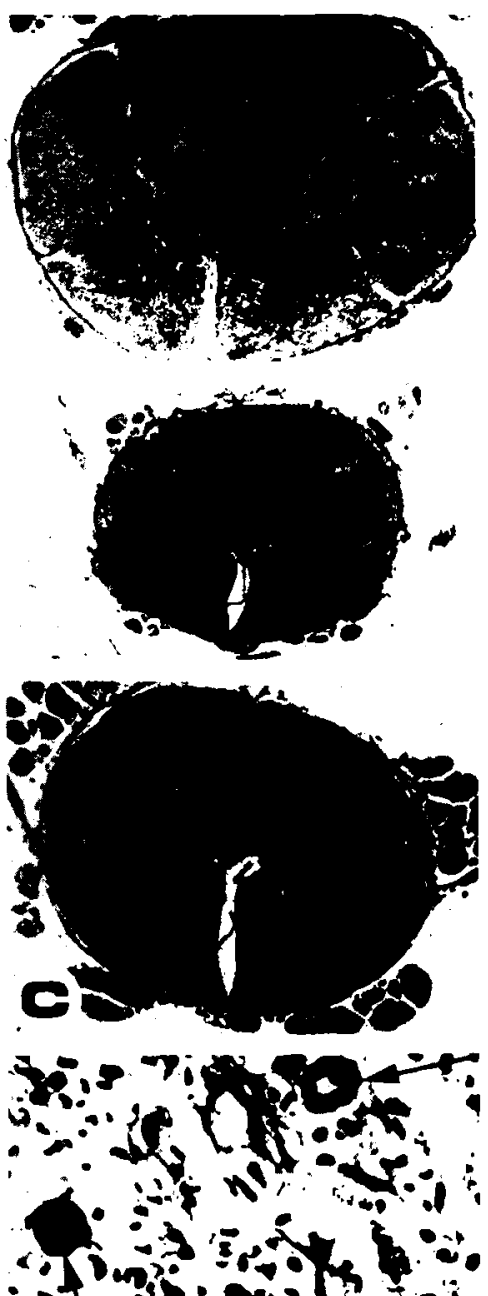

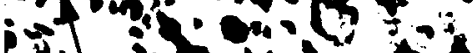
-

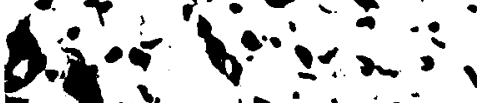

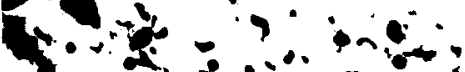
$y$ tin

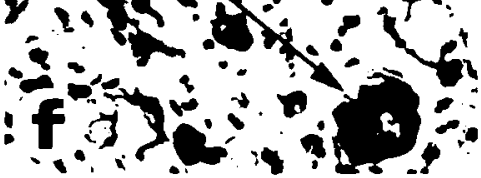

Abb. 2 a) Großer, periventrikulärer Entmarkungsherd (offener Pfeil), z.T. mit Axonschädigung, in der Umgebung des Hinterhornes, auf einem parieto-okzipitalen Hemisphärenschnitt (Markscheidenfärbung mit Luxol-Fast-Blue MBS); beachte die in der Peripherie des Herdes deutliche konzentrische Schichtung. b) Entmarkungsherd im mittleren Kleinhirnstiel (Pfeil). c) Querschnitte durch Hals-, Thorakal- und Lumbalmark (Luxol-Fast-Blue MBS). Beachte die subtotale Entmarkung des zudem geschwollenen Halsmarkschnittes (oben), der nur noch in den markierten Arealen Reste von Markfasern enthält. In Thorakal- und Lumbalmark dagegen intakte Verhältnisse. d) Markschattenherd mit florider Demyelinisierung im Nervus opticus; e) zum Vergleich intaktes Areal mit interstitieller ödematöser Auflockerung (Markscheidenfärbung mit SudanSchwarz B; Vergr. $\times 400$ und $\times 250$ ). f, g) Axondegeneration mit kugelförmigen Degeneraten (Pfeile), und Markfaserverluste (g) im Halsmark (Ausschnittvergrößerungen von c oben). Intakte Markfaserreste sind nur noch im rechten äußeren Bildabschnitt von $\mathrm{g}$ zu erkennen (Färbungen: Bodian in $f$, Klüver-Barrera in g; Vergr. $\times 250$ in $f, x 100$ in g) 


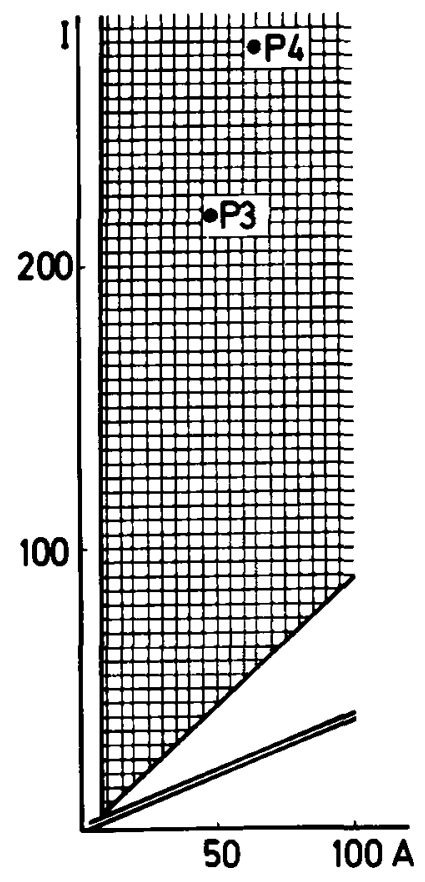

Abb. 3 Liquorprotein.Profil für Albumin und Immunglobulin G nach Reiber (29) in Fall 1 (lumbaler Liquor der Punktion (P) 3 und 4). $I=\operatorname{lgG}$ (Liquor)/lgG (Serum) $\cdot 10^{3}, A=$ Albumin (Liquor)/Al. bumin (Serum) $10^{3}$ karierter Bereich: Schrankenstörung mit zusätzlicher autochthoner IgG-Produktion

\section{Diskussion}

Klinisch wiesen unsere beiden Patienten jeweils die typische Symptomkombination der N.o. mit schweren Ausfällen im Bereich des Halsmarks sowie Neuritis optica mit erheblicher Visusbeeinträchtigung auf. Wie häufig bei der N.o. beschrieben, handelte es sich um schwere Verläufe:

Fall 1 war gekennzeichnet durch nur unvollständig remittierenden schubförmigen Verlauf mit jeweils erheblichen Restsymptomen; auffällig war das lange Intervall zwischen dem Auftreten der Neuritis optica und der Manifestation der Rückenmarkssymptome. Im allgemeinen gilt als typisch für die N.o. ein besonders stürmischer und weitgehend remissionsloser Verlauf (22). Dabei setzen in der Regel nur Tage bis Wochen nach der Sehstörung die Rückenmarkssymptome ein, als minimale und maximale Krankheitsdauer gibt Peters (22) einen Zeitraum von 5 Tagen bzw. 26 Monaten an. Cloys und Netsky (3) kommen dagegen zu dem Schluß, daß etwa die Hälfte der Patienten mit Remissionen monate- bis jahrelang überlebten. Ein Beispiel für eine lange Überlebenszeit ist auch die von Howell et al. (12) eingehend dokumentierte Patientin, bei der zwischen Erstmanifestation mit 14 Jahren und dem Tod ein Intervall von 17 Jahren lag. In unserem Fall 2 trat aufgrund der Lokalisation und Ausprägung des Rückenmarksprozesses beim zweiten Schub nach ebenfalls mangelhafter Remission des ersten bereits 6 Monate nach Erkrankungsbeginn der Exitus ein.

Der neuropathologische Befund ergab in Fall 2 schwere Nekrosen im Rückenmark neben Plaques im Großhirn; in Fall 1 zeigte sich computertomographisch ein Areal mit deutlicher
Dichteminderung im Halsmarkbereich. Beide Fälle wiesen somit morphologische Merkmale auf, die über das bei klassischer multipler Sklerose zu beobachtende Bild hinausgehen; folgt man der Klassifikation Oppenheimers (21), wäre Fall 1 in die erste oder zweite, Fall 2 in die zweite Kategorie der N.o. einzuordnen.

Entsprechend dem schweren neuropathologischen bzw. computertomographischen Befund mit ausgedehnter Nekrose wiesen unsere Fälle deutliche pathologische Liquorbefunde mit massiver Eiweißvermehrung auf. Eine ähnliche Erhöhung des Gesamtproteins im Liquor wurde ebenfalls von Howell et al. (12) und Kohut und Richter (13) in ihren Fällen von N.o. beschrieben. Die Vermehrung der Globulinfraktion bei Fall 1 mit erhöhtem IgG und einem mit 0,36 mehr als zweifach über der Norm liegenden IgG-Gesamtproteinquotienten ähnelt qualitativ - aber nicht quantitativ! - den bei der MS beschriebenen Befunden und weist auf das Vorliegen eines schweren entzündlichen demyelinisierenden Prozesses hin; daß gleichzeitig der IgG-Wert im Serum leicht erniedrigt war, spricht dafür, daß auch in unserem Fall - wie es für die klassische MS mehrfach bewiesen wurde (32) eine erhebliche lokale IgG-Synthese im ZNS stattgefunden hat ${ }^{1}$. Das Ausmaß der Eiweißerhöhung im Liquor mit deutlicher Vermehrung auch der Albuminfraktion weist auf der anderen Seite darauf hin, daß ein weiterer wichtiger Mechanismus der Übertritt von Proteinen in den Liquor im Bereich der Nekrose bei hier entsprechend gestörter Bluthirnschranke ist (Abb. 3). Diese Annahme wird dadurch gestützt, daß die im Liquor besonders deutlich erhöhten IgA- und IgM-Fraktionen auch im Serum eine Erhöhung auf das Doppelte der Durchschnittswerte bei Normalpersonen aufwiesen. Dabei bewegte sich der IgM-Wert im Serum in einem Bereich, der von Patienten mit MS gerade noch erreicht werden kann (Durchschnittswert $202 \pm 94 \mathrm{mg} \%$ bei Delmotte und Demianski (4); der IgA-Wert im Serum war dagegen eindeutig höher als bei Frauen dieser Altersklasse, die unter einer MS litten, beobachtet wurde (4). In unserem Fall 2 erfolgte nur bei der ersten Lumbalpunktion eine Bestimmung der Immunglobuline, die eine leichte IgA-Vermehrung zeigte; nach der Verlegung wurde bei den weiteren Liquoruntersuchungen, die dann ja eine deutliche Eiweißvermehrung zeigten, leider auf eine Differenzierung der Immunglobuline verzichtet.

Der Befund in unserem Fall 1 verdient besondere Beachtung, beschrieben doch auch Howell et al. (12) bei ihrer Patientin eine erhöhte IgA-Fraktion in Serum und Liquor. Bei Fällen mit typischer MS wurden mit Hilfe der verfeinerten Nachweismethoden in den letzten Jahren IgA- und IgM-Erhöhungen im Liquor gefunden $(19,20,31,34)$, doch waren die Änderungen quantitativ nicht annähernd so ausgeprägt wie in unserem Fall 1, bei dem der IgA-Gesamtproteinquotient bei 0,08 , der entsprechende Wert für IgM bei 0,04 lag. Parallel zur Eiweißerhöhung wies unser Fall 1 hohe Zellzahlen im Liquor auf, wie sie in diesem Ausmaß bisher nur von Popow (24) mitge teilt wurden. Bei Fällen mit multipler Sklerose werden entsprechende Gesamtprotein- und Zellwerte im Liquor nicht beschrieben $(16,25,26,31)$. Daß manche Autoren keinen Unterschied der Liquorbefunde zwischen MS

\footnotetext{
${ }^{1}$ Nach der von Tourtelotte et al. (33) angegebenen Formel wurden Werte von $979 \mathrm{mg}$ (Punktion 3) bzw. $804 \mathrm{mg}$ (Punktion 4) für das täglich de novo im ZNS synthetisierte IgG errechnet
} 
und N.o. sehen, liegt wahrscheinlich darin begründet, daß sie unter dem Syndrom der N.o. ausschließlich oder zumindest ganz überwiegend Patienten beschrieben, die keine Nekrose des ZNS aufwiesen, nach der Klassifikation Oppenheimers (21) also dem Typ 3 der N.o. entsprächen.

$\mathrm{Ob}$ aus der Tatsache, daß bei einigen klinisch sehr schwer verlaufenden Fällen des Typs 1 und 2 hochpathologische Eiweißwerte im Liquor nachzuweisen sind, auf eine eigene nosologische Entität dieser Fälle zu schließen ist, muß zur Zeit offenbleiben. Oppenheimer (21) selbst fuhhrt hierzu aus, daß es sich bei den zugrunde liegenden Nekrosen um sekundär zirkulatorische Effekte mit Ischämie infolge Rükkenmarksschwellung bei einem primär demyelinisierenden Prozeß handeln könnte, eine Möglichkeit, die auch von Howell et al. (12) in ihrem Fall diskutiert worden war. Eine endgültige Klärung dieser Frage wird erst möglich sein, wenn die ätiopathogenetischen Mechanismen bei der MS und den Fällen mit N.o. erkannt sind.

Zusammenfassend können aus den mitgeteilten Kasuistiken folgende Schlüsse gezogen werden:

1. Ein jahrelanges Intervall zwischen den einzelnen Schüben schließt das Vorliegen einer Neuromyelitis optica nicht aus.

2. Eine massive Erhöhung der Proteine im Liquor, z.T. kombiniert mit deutlicher Zellvermehrung, zeichnet einige Fälle von Neuromyelitis optica aus; dieser Befund unterscheidet diese Fälle von Patienten mit multipler Sklerose, bei denen entsprechende hochgradige Liquorveränderungen nicht gefunden werden. Hauptursache der massiven Eiweiß- und Zellvermehrung im Liquor dürfte die Nekrose im Rückenmarksbereich sein.

3. Ob die Rückenmarksnekrose Resultat einer Ischämie bei Rückenmarkschwellung infolge eines wie auch immer gearteten entzündlichen Prozesses im Sinne einer akuten multiplen Sklerose oder Ausdruck einer eigenen nosologischen Entität ist, läßt sich zum jetzigen Zeitpunkt, auch unter Berücksichtigung neuropathologischer Befunde, nicht schlüssig beantworten.

4. Unser Fall 1 sowie die in mancher Hinsicht ähnliche Patientin von Howell et al. (12) wiesen außer im Liquor auch im Serum erhöhte IgA-Werte auf, wie sie bei Fällen mit MS nicht gefunden wurden. Möglicherweise ergeben sich hier Ansatzpunkte zu einer pathogenetischen Abgrenzung gewisser Formen der N.o. gegenüber der MS.

\section{Literatur}

1 Alajouanine, $T ., T$. Homet, $R$. Thurel, R. Rossano: Un cas anatomo-clinique de sclérose en plaques aigue avec symptomatologie de neuro-opticomyélite. Rev. neurol. 64 (1935) 98

2 Balser, B.H.: Neuromyelitis optica. Brain 59 (1936) 353

3 Cloys, D.E., M.G. Netsky: Neuromyelitis optica. In: P.J. Vinken and G.W. Bruyn, eds.: Handbook of clinical neurology. Vol. 9, North Holland Publishing Co., Amsterdam 1970

4 Delmotte, P., L. Demonty: Biochemical findings in multiple sclerosis. II. A detailed study of the serum IgA and IgM levels of $772 \mathrm{MS}$ patients compared with 226 neurological controls. J. Neurol. 211 (1976) 135

5 Devic, A.: Myélite subaigue compliqué de névrite optique. Bull. méd. (Paris) 8 (1894) 1033

6 Ferraro, A.: Pathology of demyelinating diseases as an allergic reaction of the brain. Arch. Neurol. (Chic.) 52 (1944) 443
7 Gault, F.: De la neuromyélite optique aigue. Thése de Lyon 1894

8 Goulden, $C_{.}$: Optic neuritis and myelitis. Trans, ophthal. Soc. U.K. 34 (1914) 229

9 Hallervorden, J.: Die zentralen Entmarkungskrankheiten. Dtsch. Z. Nervenheilk. 150 (1940) 201

10 Hassin, G.B.: Neuroptic myelitis versus multiple sclerosis. Arch. Neurol. (Chic.) 37 (1937) 1083

11 Hill, Z.R.: Some aspects of neuromyelitis optica. Trans Ophthalm Soc. U. Kingd. 58 (1938) 144

12 Howell, D.A., E.H. Jellinek, K. Gavrilescu: Demyelinating disease in a woman from tropical South America with features of multiple sclerosis and neuromyelitis optica. Clinical, protein chemistry and pathological findings. J. neurol. Sci. 7 (1968) 115

13 Kohut, H., R.B. Richter: Neuro-optic myelitis, J. Nerv. Dis. 101 (1945) 99

14 Lumsden, C.E.: Fundamental problems in the pathology of multiple sclerosis and allied demyelinating diseases. Brit. Med. J. 1 (1951) 1035

15 McAlpine, D.: Familial neuromyelitis optica. Brain 61 (1938) 430

16 McAlpine, D., C.E. Lumsden, E.D. Acheson: Multiple sclerosis: A Reappraisal. Churchill Livingstone, Edinburgh-London, 1965

17 Michaux, L.: La neuromyélité optique aigue. Thèse de Paris. Amette 1930

18 Miller, H.G., M.J. Evans: Prognosis in acute disseminated encephalomyelitis with a note on neuromyelitis optica. Quart. J. Med. 22 (1953) 347

19 Minigioli, E.S., W. Strober, W.W. Tourtellotte, J.N. Whitaker D.E. McFarlin: Quantitation of IgG, IgA and IgM in the CSF by radioimmunoassay. Neurology 28 (1978) 991

20 Nerenberg, S. T., R. Prasad, M.E. Rothman: Cerebrospinal fluid IgG, IgA, IgM, IgD, and IgE levels in central nervous system disorders. Neurology 28 (1978) 988

21 Oppenheimer, D.R.: Demyelinating diseases. In: W. Balckwood, and J.A.N. Corsellis, Eds.: Greenfield's neuropathology. Edward Arnold, London 1976

22 Peters, G.: Neuromyelitis optica. In: O. Lubarsch F. Henke, R. Rössle (Hrsgb.): Handbuch der speziellen pathologischen Anatomie und Histologie. Band 1II/2 A Berlin, Göttingen, Heidelberg: Springer 1958

23 Pette, $H$.: Die akut entzündlichen Erkrankungen des Nervensystems. Leipzig: Thieme 1942

24 Popow, N.: Neuromyelitis optica acuta. Dtsch. Z. Nervenheilk. 135 (1935) 142

25 Poser, S.: Multiple sclerosis. Berlin-Heidelberg-New York: Springer 1978

26 Poser, S., G. Ritter: Multiple Sklerose in Forschung, Klinik und Praxis. Stuttgart-New York; Schattauer 1980

27 Putnam, T.J., F.M. Forster: Neuromyelit is optica, its relation to multiple sclerosis. Trans. Amer. Neur. A. 68 (1942) 20

28 Redlich, E.: Uber abortive Formen der Encephalomyelitis disseminata. Dtsch. med. Wschr. I (1929) 516

29 Reiber, $H_{\text {. }}$ : Eine aktuelle Darstellung des Liquorproteinprofils zur Differentialdiagnose von Schrankenfunktionsstörungen und entzündlichen Prozessen des Zentalnervensystems. akt. neurol. 7 (1980) 127

30 Scheid, W.: Lehrbuch der Neurologie, 4. Aufl. Stuttgart - New York: Thieme 1980

31 Schliep, G., K. Felgenhauer: Serum-CSF protein gradients, the blood-CSF barrier and local immune response. J. Neurol. 218 (1978) 77

32 Tourtelotte, W.W.: Cerebrospinal fluid in multiple sclerosis In: P.J. Vinken and G.W. Bruyn, Eds.: Handbook of clinical neurology, Vol. 9. North Holland Publishing Co., Amsterdam 1970

33 Tourtelotte, W.W., A.R. Potvin, J.O. Fleming, K.N. Murthy, J. Levy, K. Syndulko, J.H. Potvin: Multiple sclerosis measurement and validation of central nervous system IgG synthesis rate. Neurology 30 (1980) 240

34 Traugott, $U_{.}$: T and B lymphocytes in the cerebrospinal fluid of various neurological diseases.J. Neurol. 219 (1978) 185 